\title{
COVID19 War, Triangle with Inflammation and Infection
}

\section{Hedayati Ch $\mathrm{M}^{1}$ and Badlou $\mathrm{BA}^{2 *}$}

${ }^{1}$ Department of Microbiology, Guilan University of Medical Sciences, Iran

${ }^{2}$ Research and Development Department, BBAdvies and Research, The Netherlands

*Corresponding author: Bahram Alamdary Badlou, Research and Development Department, BBAdvies and Research, Zeist, The Netherlands, Tel: +31302211328; Email: bbadlou@casema.nl

\section{Editorial}

Volume 4 Issue 1

Received Date: May 20, 2020

Published Date: May 25, 2020

DOI: $10.23880 /$ hij-16000161

\section{Editorial}

Worldwide all countries are suffering from the COVID19 attack with unexpected death rate, which the exact mechanism how systemic blood and microbiota responding is not elucidated completely. Previously, is highlighted more in details about 'death triangle' machinery concept over 'how cancer-microorganism-platelet(CMP)-triangle' machinery in a bi-directional complex interaction might increase risks of premature death, and in-hospital morbidity and mortality [1].

Here we are going to highlight more in details about COVID19-Infection-Inflammation (C19-I-I) triangle 'how the COVID19 in peak of triangle, in a bi-directional manner might communicate with both Infection- Inflammation processes as an angle of triangle, which are emerging from series of interaction, which we emphasized previously [1-3]. Human microbiota are inter- and external localized bacteria, fungi (including yeasts), protozoa and viruses, in different anatomical organs of the body. Some of the microbiota produced remain persistent and others are not-persistent, which could being produced and/or destructed/ cleared (patho-) physiologically.

Obviously, the COVID19 can enter subjects' body via both pathways either directly or indirectly. In a direct pathway it passes through eyes, respiratory system or mucosal membranes using (small and/or big) aerosolized particles. This kind of entrance could be affected by an exposure to Gram-negative-bacterial-released lipopolysaccharide (LPS). The first in-vivo demonstration of synergy between respiratory viruses and LPS introduced was proposed by Kristien van Reeth et al 2000 who suggested that there is a synergistic interaction /collaboration between respiratory viruses and endotoxin LPS released form bacteria [4].

In an indirect pathway COVID19 might contaminate hosts' largest organ namely skin, inconspicuously. Nevertheless, there is an potential link between COVID19 - and infection angel that might is governing by COVID19 mutations. Infection with COVID19 (speculatively) predispose human body to bacterial or fungal infections, however [4-6]. Mixed infections could be a disturbing outcome for people who have been infected with the virus via affecting body immune response or change in microbiome population.

Post-entrance COVID19 could resultina proinflammatory response and dynamic reactions. Raising evidences suggest that the presence of COVID19 in human body increases (pro-) inflammatory cytokines like IL-6 or IL-8. Increasing the level of the (pro-)inflammatory cytokines is a doubleedged blade $[5,6]$. In one hand, an increase in cytokines release has the potential to cause an increased risk of toxic shock. In the other hand, can lead to immunologic tolerance, however. Presumably, people with chronic inflammation are more likely to have a defect in the normal functioning of their cells and organs. One might speculate that because of the presence of the COVID19 virus, subsequently some of the immune system-related genes could become "on and/ or off", permanently.

In initiation phase of entrance, maybe inflammation all processes and/or at least one of all 4 features i.e. rubor (redness), calor (increased heat), tumor (swelling), dolor (pain), increased permeability, and functio laesa (loss of function) is necessary to predispose infection of COVID19. After entrance and colonization of COVID19 in the infected target-tissue and/or cells for further propagation and metastasis to another tissue and cells, the COVID19 (mutants) needs again induce a proinflammatory reaction and change in subcellular components for exocytosis and metastasis to neighboring cells, however.

The consequence of a bidirectional communication and unknown interactions between different angels i.e. 


\section{Haematology International Journal}

'COVID19-infection', and/or "COVID19-inflammation" angel of the triangle makes diagnosis and treatment more complicated; because of either unpredictable changes in the rhythm of immune-modulation prior- and/or after COVID19 entrance and colonization or biological variabilities between patients.

Besides, host's Brain-Gut-Heart regulates microbiota/ immunological responses-axis, which also determines microbiota hemostasis, and vice versa. Likewise, microbiota combination changes might affect hormonal, immunological reaction, and responsiveness against viral pathogens i.e. COVID19 and HCovs. Moreover, after the COVID19 enters subject's body, it might change the balance of the microbiota mixture, sooner or later. Consecutively, any changes in (human-)microbiota's count and combination could cause a beneficial or detrimental effect to subject's Brain-Gut-Heart (ir-)responsiveness, which regulates immune (ir-)reactions, which are important in the case of viral virulence potency during colonization [7]. Aforementioned change may lead to change in any route of human immunology include: regional, humoral and cellular responses, eventually.

The unfolded protein response (UPR) machinery is involved in all 3 abovementioned processes and regulates all processes via premature apoptosis and /or autophagy, gene regulation, cell fate $[2,3,5]$. To better understand these correlation/ association/ signal transduction/ connections for an assessment for better diagnosis and treatment, Medici need a detection system that can measure and study in-details whole C19-I-I-triangle. Although, One might hypothesize that (epi-)genetical changes might modify the UPR machinery, which (in-)directly modulate the key regulatory genes, which are involved in infection and inflammation after/ during the exposure to the COVID19 virus.

The UPR is a system could change or regulate five different processes i.e.

a) The cells and organs fate;

b) Signal transduction induction that being easily affected by physico-chemical variables of human cellular microenvironment, infections [2];

c) Could establish a bridge of communication after/ during different treatments against infections and/or inflammations. Subsequently

d) the UPR machinery might alter the various gene expression that are involved in protein synthesis, translation, transcription, or replication after tissue and cells are inflamed or infected, post-COVID19; and The UPR machinery can act as a switch control to reset deadly outcomes of endotoxemia in response to LPS over-release, in systemic blood circulation [8].

The take home message is that after entrance of COVID19, initiation processes is inflammation, which could facilitate infecting processes with (un-)predictable changes in microbiota combination, which make cells, tissues suitable for COVID19 propagation inter-and intracellularly. When COVID19 mutants are established inter- and /or intracellularly, they need to proliferate and differentiate in a metastatic aggressive way, which might again need (pro-)inflammatory cytokines storm to metastasis, and start random shut down of infected host. The complexity of COVID19-war make it so multifaceted that more in details investigation needed globally.

\section{References}

1. Badlou BA (2019) Death Triangle Machinery has Three Phases of Action. Acta Scientific Cancer 3(5): 27-28.

2. Hedayati Ch, Abedinzade $\mathrm{M}$, Golshekan $\mathrm{M}$, Badlou BA (2020) Combination of Calcium Channels Inhibitors Prevents LPS - Induced (pro-) Inflammatory Release of the TNF-a and IL-1ß, In Vivo. EC Cardiology 7(3): 1-9.

3. Badlou BA, Hedayati ChM (2020) Highlights About Recent Sepsis Related Studies. CPQ Medicine 8(4): 1-3.

4. Van Reeth K, Nauwynck H, Pensaert M (2000) A potential role for tumour necrosis factor-Alpha in synergy between porcine respiratory coronavirus and bacterial lipopolysaccharide in the induction of respiratory disease in pigs. J Med Microbiol 49(7): 613-620.

5. Sing Fung T, Liao Y, Xiang Liu D (2016) Regulation of Stress Responses and Translational Control by Coronavirus, Viruses 8(7): 184.

6. Zhang X, Yun Tan, Yun Ling, Gang Lu, Feng Liu Viral and host factors related to the clinic outcome of COVID19. Nature

7. Bandoro C, Runstudler J (2017) Bacterial Lipopolysaccharide Destabilizes Influenza Viruses. ASM 2(5): 267-217.

8. Banerjee A, Czinn S (2020) Crosstalk between endoplasmic reticulum stress and anti-viral activities: A novel therapeutic target for COVID19. Life sciences 2020: 117842 . 